\title{
Contracting for quality or local fixes? Two Personal Medical Services pilots
}

Stephen Abbott Public Health and Primary Care Unit, School of Nursing and Midwifery, City University, London, UK

\begin{abstract}
As an alternative to the national contract with general practitioners (GPs), Personal Medical Services (PMS) is a way of contracting with GPs for providing primary care, intended to enable individual contracts with practices which are appropriate to the specific needs of their populations. The national plan for the NHS (2000) strongly supports the extension of PMS as a means of improving the quality of primary care. This paper reports two small pilots which found PMS a useful mechanism for achieving desired changes within the practices. In one case, the GPs are now employed by the local community trust and the primary health care team has been expanded, with some clinical functions redistributed among the team. In the other, the primary health care teams of two practices have developed a tool for assessing the health and needs of older people during a home visit. Participants in both pilots expressed satisfaction with the results, although there were some dissenting voices. In both cases, the pilots were unable to demonstrate in detail any improvements in patient care or to quantify the work-load implications of the changes. Perhaps because of their 'pilot' status, the initiatives tended to be inward looking, and opportunities for learning across the local health economy have been missed. PMS' value as a driver for improving the quality of primary care is not demonstrated by these two examples.
\end{abstract}

Key words: older people; Personal Medical Services; primary care; salaried GPs; screening; skill mix

\section{Introduction}

In 1996, a British government White Paper, Choice and Opportunity (Secretary of State for Health, 1996) announced the government's intention of enabling local changes to the national contract with general practitioners (GPs) working within the National Health Service (NHS). The announcement reflected the government's awareness that the national legislative and contractual framework for primary care (General Medical Services (GMS)) discouraged rather than facilitated the pursuit of specifically local aims. Whereas GMS offers a complex range of payments, including both a payment for each patient registered with the GP and payments for nationally specified interventions

Address for correspondence: Stephen Abbott, Public Health and Primary Care Unit, School of Nursing and Midwifery, City University, Philpot Street, London E1 2EA, UK. Email: s.j.abbott@city.ac.uk (e.g., screening), as well as payments for premises and staff costs, PMS offers a single contract sum for the delivery of care appropriate to the particular needs of the registered population. PMS is thus theoretically able to remunerate adequately those GPs whose workloads include the care of untypically high numbers of patients with particular needs, such as older or homeless people.

It was envisaged that such changes would have one or more of three broad aims:

- to promote consistently high quality services across the country;

- to provide opportunities and incentives for primary care professionals to use their skills to the full;

- to provide more flexible employment opportunities in primary care.

However, it was emphasized that the policy was designed to facilitate local innovation and improve local responsiveness to need, rather than imposing 
a model of service organization or delivery. Crucially, such proposals were entirely voluntary, and there was no expectation that all practices could or would consider the PMS option. Thus it was unclear how this mechanism would promote national consistency.

Broadly, this policy initiative survived several changes of name and a change of government, although the criteria for acceptance for proposals were tightened by the new Labour Government to exclude schemes which focused on the commissioning of secondary care, and those which involved private sector organizations. Eighty-seven first wave Personal Medical Services (PMS) pilots began in 1998 (Leese et al., 1999). Rather larger second and third waves followed in 1999 and 2001.

Meanwhile, in 1997, the new Labour Government had announced the creation of primary care groups and trusts (PCG/Ts), which for the first time created primary care organizations with a common structure throughout England (Secretary of State for Health, 1997). This attempt to bring coherence to the diversity and fragmentation of primary care appeared to point in the opposite policy direction from that of PMS pilots, which were described as 'a thousand flowers in bloom' (NHS Executive, 1998).

The NHS national plan (Secretary of State for Health, 2000) reaffirms both of these policies. Primary care groups and trusts are the major structural arrangement of primary care in England, while at the same time, the government wishes to see considerable extension of PMS arrangements. The plan anticipates that PMS, which henceforth will be a permanent rather than a pilot arrangement where it is introduced, will cover nearly a third of general practices by 2002. To save bureaucracy, a core PMS contract has been drawn up for use nationally, although the intention is that contracts will include components which reflect local needs and conditions.

This extension of PMS is perhaps surprising, as ongoing national evaluations of first-wave pilots have reported slow progress (Walsh et al., 2000a; Steiner, 2000). It is therefore difficult to say yet whether it is a useful mechanism for improving primary care. However, the emphasis it receives in the national plan indicates that the government wishes to use it as a tool for improving quality and addressing inequalities in access to primary health care. It is interesting that PMS began as a mech- anism to allow local experiments, but has now become part of the government's attempt to assure quality and equity of health care nationally. The success or failure of PMS can therefore be judged in two rather different ways. First, has PMS been a useful and/or necessary mechanism for achieving the pilots' own objectives? Second, has PMS helped to improve the quality and equity of primary healthy care?

This paper uses data from two case studies of first-wave pilots to consider both of these questions. The pilots studied were situated in one health district in the north west of England, and the evaluation was commissioned by the local health authority, which requested a final report by the end of 2000 , so that the findings could inform decisions about PMS in the district in 2001 and beyond.

First, the pilots and the evaluation methods are described briefly, and findings are then presented for each site. A discussion section then considers whether the pilots have (1) achieved their local objectives; (2) improved the quality of primary care; and (3) helped local NHS organizations to learn more about primary care? As neither pilot sought primarily to address issues of equity of access, this aspect of PMS is not included.

\section{The PMS pilots}

The first pilot was situated in a small practice (two full-time GPs) in a prosperous small town (hereafter known as Pineville) near to a deprived metropolitan area. Its chief objective was to increase the capacity and skill mix of the primary care team, and it set out to do this by:

- changes in employment arrangements, whereby both GPs and all the previously practiceemployed staff became employees of the local community trust;

- conversion of the premises to accommodate district nursing and health visiting staff, and allow closer co-operation between them and practice staff;

- introduction of a new information technology (IT) system;

- enlargement of and skill mixing within the primary health care team (PHCT).

Prior to PMS, the practice was unable to afford the building work or the new IT system, which 
were paid for by the trust when it took over the management of the practice (it rents the premises from the GPs). It was envisaged that these mechanisms would allow the practice nurse more time to improve chronic disease management in the practice, and that skill mixing would enable the GPs to generate practice income by undertaking work elsewhere, thereby funding the extension of the team.

The second pilot was situated in a seaside town (hereafter known as Sandside), with a significant retired population, and 'bed-and-breakfast' accommodation for people without a permanent home. The pilot comprised two practices with high proportions of older people in their practice populations. The practices were not linked other than by the pilot. They were both fairly small, with one full-time and two part-time GPs in one, and two part-time GPs in the other. The pilot's aim was to enhance the primary and community care provided to older patients, and to reduce the number of admissions of older people to hospital and nursing home care. It set out to do this by:

- developing a holistic assessment tool for older people to be used during a home visit, as an alternative to the existing screening of those aged 75 years and over, and as a basis for preventive health care;

- improving clinical practice (e.g., increased audit, developing protocols for good practice, reviewing polypharmacy);

- establishing closer links with social services and the voluntary sector.

The two pilots, which are in the same PCG, are thus very different in their nature. Pineville employs salaried GPs in a single practice, aiming to improve primary care delivery in general. Sandside links two practices with the intention of improving the primary care of a specific population group. Thus they illustrate the diversity which was intrinsic to the vision set out in Choice and Opportunity.

\section{The evaluation}

The evaluations were primarily qualitative, and set out:

- to track the progress of the pilots;
- to understand how and why they developed as they did; and

- to assess the importance of their achievements.

A separate quantitative component of the research, carried out in collaboration with the national evaluation of first-wave PMS pilots (National Evaluation, 2000) will attempt to detect any changes in the quality of care which might be associated with the pilot, but this work is not complete at the time of writing.

Semi-structured interviews were conducted with key players at six-monthly intervals between summer 1998 and summer 2000. Informants were asked to give their own account of progress to date, and their views of benefits and challenges related to the pilot. Sixty interviews were carried out in Pineville, and 46 in Sandside: 26 of these were with GPs, 28 with other primary health care team (PHCT) members, and 24 with other practice staff (administration, management, receptionists, etc.). Others included health authority and social services representatives, and in the case of Pineville, staff from the community NHS trust. Interviews were taped and transcribed and analysed thematically.

\section{Findings}

\section{The Pineville pilot}

The great contrasts in organizational culture between general practice and NHS community trusts meant that it took some time before a stable and satisfactory structural relationship was established. Neither hierarchical nor small-business models of working were suited to the PMS pilot. In any case, as the pilot was being set up, the community trust was also preparing to merge with a neighbouring trust. The pilot eventually adopted a 'self-managed' model: a core group of the PHCT, led by a project co-ordinator, took executive control of the practice on a day-to-day basis, while a trust manager acted as project manager and was the main operational link between the pilot and the trust's management and resource infrastructure.

The project co-ordinator role was originally carried out by a GP, and was then passed to the practice manager so that the GP could devote more time to clinical work. When the practice manager left, the role returned to the same GP, perhaps 
surprisingly, given that the opportunity costs of highly qualified clinicians taking on administrative/ management roles are necessarily high.

Effective links between the practice and the trust took time to develop, not least because the person providing the main practice/trust link changed several times before and during the merger. The team struggled to manage itself efficiently, being dependent on the trust for information and resources. Operational problems included cumbersome systems for obtaining supplies and arranging repairs, accounting errors, and delays in providing budgetary information. Relationships between the pilot and the community trust significantly improved as the trust's investment of management time increased and became more consistent.

Overall, there was considerable stress on staff at the beginning of the project. Multiple changes in the office/reception staff team, particularly in the first year, were very disruptive, and some new staff appeared to receive insufficient training. There was some expressed dissatisfaction among patients about the practice's efficiency in organizing appointments, repeat prescriptions, etc.

There were changes in the skill mix of the PHCT at Pineville: some nursing members took on new roles, and the nursing team expanded. As a result, the practice nurse undertook more chronic disease management, a community nurse provided wellwoman and family planning services, and by the third year, the GPs each worked one and a half days per week outside the practice. However, staff turnover meant that progress in improving chronic disease management was much slower than expected.

Some team members said that the PHCT was working better together as a result of more frequent meetings and because community nurses were accommodated on the premises. However, unresolved tensions between practice and community nurses persisted, despite significant team-building input from trust personnel. Difficult team dynamics were thought by some to have contributed to staff turnover. Also, there appeared to be confusion within the PHCT about how individual, professional and team management and responsibilities mesh together, and no sense of corporate and collective accountability was apparent.

The expansion of the PHCT increased patient access to nursing care. There were also some reductions in access, due to slightly shorter open- ing hours, and by 2000, significant reductions in the numbers of appointments available to patients with GPs. Some patients expressed resentment to reception staff at not being able to obtain appointments or repeat prescriptions as promptly as before.

\section{The Sandside pilot}

A new assessment tool was developed by the pilot, embodying a holistic approach to the health and social care needs of older people. It was designed to be used during a home visit, to capture a wider range of information than did previous screening assessments of those aged 75 years and over, and to identify those patients at risk of accidents and/or of rapid deterioration in their health. It was used primarily by nursing staff. Patients were selected for assessment according to set criteria: greater age, living alone, taking several medications. Assessments were also carried out opportunistically, during other contacts with patients (e.g., 'flu injection).

Most older patients registered with the practices were assessed. The assessments uncovered relatively little unmet need for health care, but some unmet need for social support (relating to isolation, anxiety about money, etc.) and for preventive care (e.g., smoke alarms). Staff reported that assessed patients appreciated the time and interest taken by professionals and the fact that a home visit took place. Staff enjoyed and valued the assessment process, and believed that the multidisciplinary discussions of assessed patients at each practice improved the teamwork in each PHCT, and ensured a thorough review of patients' care.

However, a number of weaknesses can be identified in the process whereby the assessment tool was developed. The pilot chose to develop its own assessment tool in-house rather than to use existing tools, and the resulting product was not validated. (A limited exercise in which two PHCT members visited a patient together and completed separate assessment forms showed a significant number of differences in how the forms were completed.) No project policy was formulated to guide how often reassessments should take place, or how patients assessed to be at risk should be monitored and supported over time. A postal questionnaire was devised for annual follow-up with patients not initially assessed to be at risk, which was used only to a limited degree, and not validated. 
The impacts on individual and population health, and on professionals' workload, were not monitored. It is therefore not known whether benefits for patients were commensurate with the considerable time and effort required by the assessment process.

The pilot tended not to look outward to its local environment. For example, similar work on assessment was being undertaken elsewhere in the same town, duplicating the pilot's work to some extent, but attempts to make links between these parallel pieces of work were made only latterly. The pilot's focus remained on improving primary care, a valuable aim in itself but perhaps not the best use of the pilot's development money, given the consensus view that the pilot practices already offered a high standard of care to older people. One of the pilot's broader aims, to reduce levels of hospital and nursing home care of older people, was not attempted beyond the hope that the assessment process might enable the PHCT to help patients avoid accidents or morbidity which in turn might reduce hospital admissions.

There were difficulties in recruiting and retaining a project manager in the first year, which the teams found frustrating. However, the experience of the two people who did take on the role in the second and third years, respectively, was that there was some reluctance within the teams to support the leadership aspect of the role. Indeed, more was achieved in the first year than in subsequent years, suggesting that perhaps a project manager role was not suitable for a project involving two separate teams of relatively autonomous clinicians who were not seeking to work more flexibly.

The two practices were quite separate except for the PMS pilot, and did not even share a community nursing team. Moreover, only one of the five GPs in the two practices worked there full time. A priori, these might have been expected to be cumbersome arrangements for joint working, and in reality, the practices tended to work separately and in parallel, rather than together. Project managers believed that more co-operation would have added value by sharing learning and experience and by reducing duplication of effort.

Some informants spoke of the considerable effort which had gone into developing and updating the PMS contract between the GPs and the health authority. There were concerns that the health authority had not fully understood the com- plexities of GP payments, and there were delays in arranging for PMS contracts to reflect inflationary rises in GMS (as elsewhere (Walsh et al., 2000a)). Though the PMS contract had some advantages for GPs, particularly regular and predictable payments, these have to be balanced against high transaction costs.

\section{Discussion}

Case studies are a particularly suitable research method when "both the "case" and its context may be changing over time' (Yin, 1999). Such change over time did indeed characterize the context in which these examples developed, in particular the preparation for and creation of PCG/Ts. The data reported here illustrate some of the strengths and weaknesses of PMS in practice, in relation to the three questions posed in the introduction, although generalizations about PMS as a whole cannot be derived from just two first-wave examples.

\section{Was PMS a useful and/or necessary mechanism for achieving the pilots' objectives?}

These case studies offer rather mixed evidence of the value of PMS in achieving objectives: PMS did have a distinctive role to play in Pineville, but not at Sandside. However, all three practices were able to use it to ease the cost and time pressures associated with new work.

Although Pineville used the PMS innovation of salaried GPs, its basic aims were not particularly innovative. The bringing together of practice and community nursing is not unusual, although it is untypical locally, while skill mixing within nursing teams is widely recognized as an important developmental task for primary care (Audit Commission, 1999; Department of Health, 1999). The expansion in nursing roles at Pineville has been limited compared with that achieved by some PMS pilots (Walsh et al., 2000a), and the integration of the team appears to have created or exacerbated conflicts. Although PMS cannot be blamed for team conflicts, which can arise under any organizational arrangement, the difficulties experienced at Pineville demonstrate the limitations of structural arrangements in facilitating better processes for good quality care.

Although integration and skill mixing in the nursing team have been achieved elsewhere with- 
out PMS, the PMS mechanisms was necessary in this instance because of the practice's financial position under GMS: PMS enabled the trust to take financial responsibility for new investment. PMS' efficacy in this case will have relevance to a number of practices where there is a need to develop both the premises and the PHCTs, particularly in single-handed practices. However, many singlehanded GPs enjoy their independence (Davies, 1998), and would not necessarily be willing to be employed by a trust.

It should be added that although originally the GPs regarded salaried status as a means to a wider end, they were both very glad to be free from the pressures of running a small business, which is consistent with the findings of the national evaluation of PMS pilots (National Evaluation, 2000).

At Sandside, the PMS contract in itself was not perceived by informants as a key component of the project. The pilot's value for most of those interviewed was the development money available to the pilot, which enabled the GPs to be remunerated for additional work, such as reviewing patient notes, attending multidisciplinary meetings, etc., and paid for some extra nursing time. The same money provided on a project basis could have produced the same results, although it is of course true that PMS pilots attracted funding which was not available to GMS practices.

Funding apart, PMS made no radical difference to how the practices ran. The practices continued to behave with the independence characteristic of general practice, rather than to explore more cooperative and flexible relationships within and outside the PHCTs. This tendency for traditional organizational forms to persist within PMS has been reported elsewhere (National Evaluation, 2000).

\section{Did PMS help to improve the quality of primary care offered?}

In practice, neither pilot demonstrated a commitment to demonstrably improving quality as the major objective, and the difficulties of monitoring quality were not effectively addressed or resolved either by themselves or by the health authority. At Sandside, the work undertaken was insufficiently grounded in existing evidence of good practice. At Pineville, on the other hand, the reduction in GP availability could be interpreted as a reduction in one aspect of quality, i.e., access, and there were some failures to meet screening targets (immunization and vaccination, cervical smear tests, etc.). Though there may have been improvements in quality in other respects, data were not systematically recorded and analysed at either pilot which could demonstrate these.

Nationally, PMS appears to be promoting more collaboration (National Evaluation, 2000), and staff from these two pilots pointed to improvements in team working. These were thought to have arisen from a perceived increase in communication, because of shared premises at Pineville and more multidisciplinary meetings at Sandside. Informants assumed that better communication was in itself an increase in quality and would result in improved patient care.

This rather weak focus on quality is not just a local phenomenon. It has already been noted that PMS contracts in general have struggled to embody adequate quality indicators (Sheaff and Lloyd-Kendall, 2000; Steiner et al., 2000). This leaves open to question the argument that PMS is a mechanism for quality assurance or improvement, as confidently asserted in the NHS National Plan (Secretary of State for Health, 2000).

\section{Has PMS helped NHS organizations to learn more about primary care?}

Practices which became first-wave PMS pilots were attempting to address their own problems and challenges (Lewis and Mays, 1999), and PMS activity was not therefore necessarily grounded in the strategic objectives of PCGs (Glendinning, 1999). It was therefore never a given that PMS in itself would facilitate shared learning and joinedup working across local health economies. General practices have traditionally tackled their task as small businesses, rather than as public sector planning organizations, and PMS only partly challenged that by offering the possibility of GPs being employed by other than primary care organizations, an opportunity taken up by 22 pilots (25\%) (National Evaluation, 2000). Those working within these two pilots described here did not make major efforts to share learning with the local NHS, except that one Pineville GP was active in trying to persuade a generally unsympathetic local GP community of the benefits of salaried status, with some success.

National evaluators of PMS have noted a tend- 
ency for pilots to focus inward (National Evaluation, 2000). This can be attributed in part to the fact that PMS pilots were developed in an environment which was dominated by PCGs, which had to be developed at great speed (Wilkin et al., 2000), and were a preoccupation for primary care and for health authorities (Lewis et al., 1999; Steiner etal., 2000; Walsh etal., 1999). As a result, PMS pilots and PCGs have coexisted rather than forging strong mutual links (Walsh et al., 2000b).

It has been argued that PMS pilots can be seen as a test bed for primary care trusts (Gillam, 1999). At Sandside, links between the PMS pilot and the community trust were only initiated in the third year, so the extent of actual learning for the future cannot yet be determined. In the case of Pineville, key personnel in the community trust did indeed recognize that managing the PMS had taught them a great deal about primary care, but as the trust becomes two PCTs in April 2000, the ability of the organization to benefit from individual learning will depend on the new roles those individuals are given. There was no sense that the learning had gone beyond that of individuals and had become embodied in the organization as a whole.

\section{Conclusion}

Given not only the faith which the government is placing in PMS, but also the considerable efforts which were required to bring about change, it is disappointing that evidence from these two examples of PMS's use as a mechanism for promoting quality and flexibility is not stronger. The Pineville and Sandside pilots illustrate the tendency for small-scale practice-based projects to look inward and to work in isolation. Some benefits may become clearer in time. For example, community trust staff involved with the Pineville pilot may be able to use their learning to good effect in the new PCTs, and the increased receptivity among local GPs to the idea of salaried status may encourage other practices to innovate in this way. Nevertheless, it is arguable that developing PMS practices or groups of practices is both resource intensive and an unhelpful distraction at a time when health authorities and PCG/Ts are still learning new functions and relationships. Such organizations will need to work together to ensure that existing and future PMS practices justify the time and opportunity costs required by their development, and that they contribute to wider learning across the local health and social care system. In this respect, it is encouraging that the health authority which hosts the two pilots discussed here has now begun working jointly with a neighbouring authority to coordinate the planning, support and monitoring of first, second and third wave pilots across both districts.

\section{References}

Audit Commission. 1999: First Assessment. A review of district nursing services in England and Wales. London: Audit Commission.

Davies, J. 1998: Going solo. Health Services Journal 1/10/98, $28-9$.

Department of Health. 1999: Making a difference. Strengthening the nursing, midwifery and health visiting contribution to health and healthcare. London: Department of Health.

Gillam, S. 1999: Does the new NHS need personal medical services pilot? British Medical Journal 318, 1302-303.

Glendinning, C. 1999: GPs and contracts: Bringing general practice into primary care. Social Policy and Administration 33, 115-31.

Leese, B., Gosden, T., Riley, A., Allen, L. and Campbell, S. 1999: Setting out. Piloting innovations in primary care. Manchester: National Primary Care Research and Development Centre.

Lewis, R. and Gillam, S (eds). 1999: Transforming primary care. Personal medical services in the new NHS. London: King's Fund Publishing.

Lewis, R., Jenkins, C. and Gillam, S. 1999: Personal Medical Services pilots in London: rewriting the Red Book. London: King's Fund Publishing.

Lewis, R. and Mays, N. 1999: Trends in Government health policy - from GMS to PMS. In: Lewis, R. and Gillam, S. (editors), Transforming Primary Care. Personal Medical Services in the new NHS. London: King's Fund Publishing.

National Evaluation of First Wave NHS Personal Medical Services Pilots. 2000: Integrated interim report from four research projects. Manchester: National Primary Care Research and Development Centre.

NHS Executive. 1998: Personal Medical Services Pilots under the NHS (Primary Care) Act 1997. A comprehensive guide. London: NHS Executive.

Secretary of State for Health. 1996: Choice and Opportunity. London: Department of Health.

Secretary of State for Health. 1997: The new NHS. Modern. Dependable. London: Department of Health.

Secretary of State for Health. 2000: The NHS Plan. A plan for investment. A plan for reform. London: Department of Health. 
Sheaff, R. and Lloyd-Kendall, A. 2000: Principal-agent relationships in general practice: the first wave of English Personal Medical Services pilot contracts. Journal of Health Services Research and Policy 5, 156-63.

Steiner, A (ed.). 2000: Does PMS improve quality of care? Interim report to the Department of Health. Manchester: National Primary Care Research and Development Centre and Southampton: University of Southampton.

Walsh, N., Allen, L., Baines, D. and Barnes, M. 1999: Taking off: a first year report of the Personal Medical Services (PMS) pilots in England. Birmingham: Health Services Management Centre.

Walsh, N., Huntington, J., Barnes, M., Rogers, H. and Baines,
D. 2000a: PMS pilots: a change for the better? Briefing Paper. Birmingham: Health Services Management Centre.

Walsh, N., Huntington, J., Barnes, M., Baines, D. and Rogers, H. 2000b: Friends and relations. Health Services Journal 10/8/00, 28-29.

Walsh, N. and Huntington, J. 2000: Testing the pilots. Nursing Times 96, 32-33.

Wilkin, D., Gillam, S. and Leese, B. 2000: The national tracker survey of primary care groups and trusts. Progress and challenges 1999/2000. Manchester: National Primary Care Research and Development Centre and London: King's Fund.

Yin, R.K. 1999: Enhancing the quality of case studies in health services research. Health Services Research 34, 1209-225. 